\title{
U-Shaped Wire Based Resonators for Viscosity and Mass Density Sensing
}

\author{
Martin Heinisch ${ }^{1}$, Erwin K. Reichel ${ }^{1}$, Bernhard Jakoby ${ }^{1}$ \\ ${ }^{1}$ Institute for Microelectronics and Microsensors, Johannes Kepler University, Altenbergerstraße 69, \\ 4040 Linz, Austria \\ martin.heinisch@jku.at
}

\begin{abstract}
In this contribution, miniaturized U-shaped resonators used for viscosity and mass density sensing are presented. These devices were especially designed to reduce and overcome spurious instabilities, which were observed in previous sensor designs. Four basic sensor designs are presented and their sensitivities to viscosity and mass density are examined. The particular setups are discussed in their working principle, accuracy, stability and repeatability. First measurements are shown and the theory necessary to relate the measured data to viscosity and mass density is outlined.
\end{abstract}

Key words: Viscosity, Mass Density, Resonators, Sensors, Kilo-Hertz Range

\section{Introduction}

Viscosity is the characteristic of a fluid to dissipate energy, when it is disturbed from its equilibrium by forces acting on the latter. [1] It is an important property for many technological and scientific applications and thus, there has been a large effort in the development for accurate and high-precision viscosity measurement setups. A large number of commercially available laboratory viscosity measuring methods and according submethods are extensively reported in literature, see e.g., [2, 3] and found many applications in fields such as food, biological, medical, chemical and automotive industry, just to name some of them. However, these mostly very expensive measuring instruments are difficult to integrate, e.g. in a production line, for inline measurements due to their relatively large overall sizes and their maintenance requirements.

When it comes to the characterization of viscoelastic liquids which exhibit a significant frequency dependence in their shear modulus (whose imaginary part is related to the shear viscosity), oscillatory measurements are performed. However, laboratory rheometers can only be operated in a limited frequency range not exceeding $\sim 100 \mathrm{~Hz}$. Miniaturized devices sensing viscosity which have also been extensively discussed in the past, e.g. thickness shear mode resonators, [4] work in the $\mathrm{MHz}$ range.
These circumstances define the motivation for miniaturized viscosity sensors which incorporate low production costs, the ability for in-line integration and hand-held applications. To allow for characterizing viscoelastic liquids as well, the sensing principle should be based on oscillatory measurements and furthermore, to close the aforementioned gap between $100 \mathrm{~Hz}$ and $1 \mathrm{MHz}$, be operated in the kilo Hertz range, which is of special interest for rheologists.

Several approaches have been pursued, to meet these framework requirements. Most recently introduced devices utilize vibrating resonant mechanical structures interacting with the fluid where their resonance frequency and their quality factor are affected by the fluid's viscosity and mass density. For actuation and read-out of these mechanical resonators, piezoelectric, piezoresistive as well as optical methods have been extensively reported in the past, see e.g., [5]. Through their advantages of long term stability, no restrictions by the optical characteristics of the examined liquids, relatively easy manufacturing as well as simple implementation of actuation and read-out electronics, Lorentz-force actuated resonators which are read-out by a motion-induced voltage, turned out to be optimally suited for miniaturized viscosity and mass density sensors. For these Lorentz-force based resonators, the investigated principles include e.g., vibrating membranes $[6,7]$, in-plane oscillating platelets $[8,9]$ and vibrating wires [10]. The latter was especially designed to allow 
for tuning the resonance frequency, enabling measurements in a given frequency range. However, the first designs for all of the aforementioned principles strongly suffered from instabilities of the resonant behavior (resonance frequency and quality factor) as well as high cross-sensitivitiy to temperature which is caused by thermally induced mechanical stresses in the resonating structures. These drawbacks enforcedly yield low repeatability and accuracy in the measurements. The implementation of meander shaped beams supporting the in-plane oscillating platelets strongly reduced their ross-sensitivity to temperature. For the case of wire-based resonators, the straight wire, which can be interpreted as a doubly clamped beam, was replaced by a U-shaped structure as it is depicted in Fig. 1. This design comes close to a singly clamped beam and thus eliminates any potential changes of (normal) stresses in the structure effecting the spurious changes in resonance frequency. In fact, the dependency of the resonance frequency to temperature could be decreased from $\sim 35 \mathrm{~Hz} / \mathrm{K}$ for first setups of the straight wire down to $\sim 0.05 \mathrm{~Hz} / \mathrm{K}$ for the U-shaped devices, see [10], and Fig. 2. In first experiments with these devices resonance frequencies from about $100 \mathrm{~Hz}$ to $40 \mathrm{kHz}$ could be obtained.

For the principle of fluid characterization by recording the frequency response of a mechanical oscillator, it is possible to determine both, viscosity and mass density with one single measurement. However, in some cases it might be beneficial or required to determine the mass density with an additional, separate measurement. For this case, a concept of a measurement cell has been developed, where viscosity and mass density sensor units are of the same size and based on the same measuring princlpe, see Fig. 1. There, Ushaped conductors carrying sinusoidal currents are placed in an external magnetic field and thus oscillating in an out-of plane mode. This oscillation is measured by the motion-induced

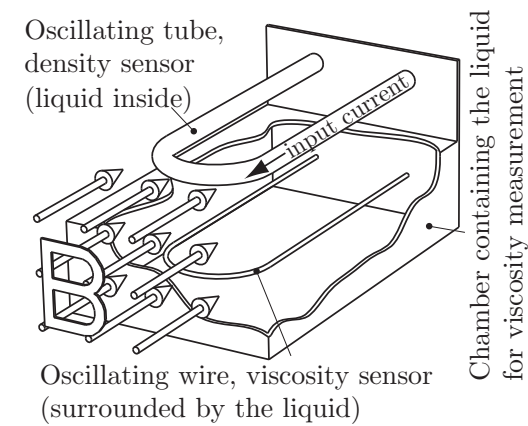

Fig. 1. Sketch of a measuring cell containing a viscosity sensor (bottom) and a mass density sensor (top) based on U-shaped cantilever beams.

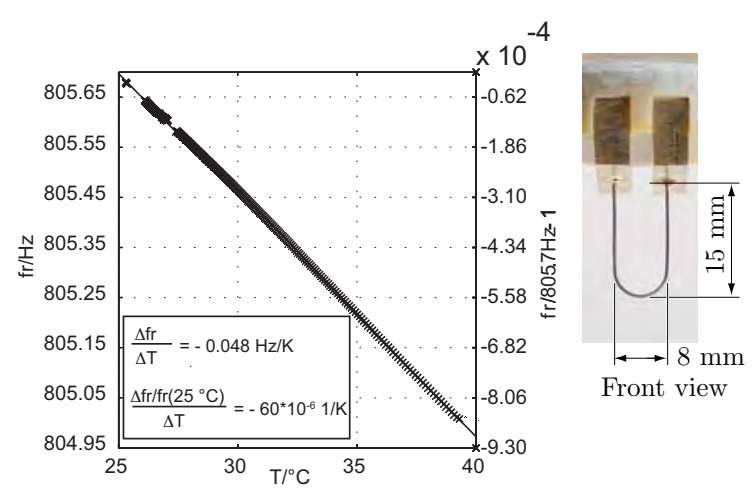

Fig. 2. Measurement: Resonance frequency over temperature for a $400 \mu \mathrm{m}$ thick tungsten wire brazed into two solid brass blocks.

voltage $V_{M}$ on the wire, (c.f electrical equivalent circuit in Fig. 3). The frequency response is recorded with a lock-in amplifier by sweeping the excitation frequency (provided by a function generator) in a range containing the fundamental (out-of-plane) mode. The recorded frequency response is then related to the viscosity and mass density of the liquid. The viscosity sensor consists of a tungsten wire which is immersed into the liquid, whereas the mass density sensor consists of stainless steel tube carrying the sample liquid inside. In general, higher viscosities and mass densities yield higher damping and lower mass densities, respectively.

In this contribution, first, a theoretical investigation for these fluid sensors is explained. There, closed form models for the Ushaped viscosity sensor and two variants of the latter (a platelet and a sphere, respectively, being attached at the U's end, see Fig. 4) as well as for the mass density sensors are presented. In the subsequent sections a new measurement setup is explained and first measurement results for the U-shaped devices are shown.

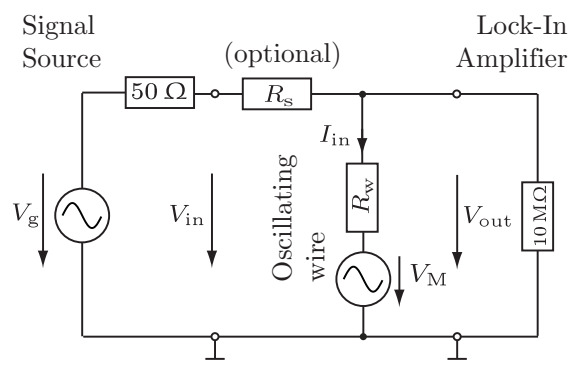

Fig. 3. Electrical equivalent circuit of the $U$-shaped wire. The series resistor $R_{s}$ is used to limit the excitation currents to prevent from non-linear deflection. $R_{w}$ is the resistance of the wire. 


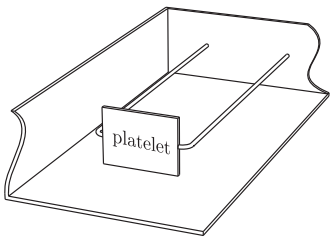

a) Viscosity Sensor with an attached platelet

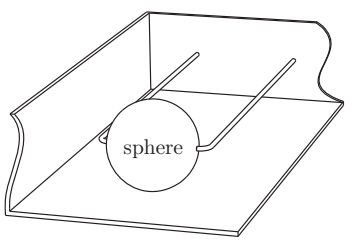

b) Viscosity Sensor with an attached sphere
Fig. 4. Variants of the viscosity sensor.

\section{Theoretical considerations}

For modeling, the U-shaped structures are modeled by a singly clamped beam (cantilever) with a lumped mass at its end, see Fig. 5. Assuming, that the flexural deflections of the $U$ shaped beam are small compared to it's length and that the effects of shear deformation may be neglected, classical Euler-Bernoulli beam theory can be used to describe the deflections $u_{z}(x, t)$ of the U-shaped structure, c.f., $[11,12]$ by the following linear, partial differential equation of fourth other:

$$
E I \frac{\partial^{4} u_{z}(x, t)}{\partial x^{4}}+m^{\prime} \frac{\partial^{2} u_{z}(x, t)}{\partial t^{2}}+d^{\prime} \frac{\partial u_{z}(x, t)}{\partial t}=0
$$

( $E$ : Young's modulus, l: second moment of area, $x$ : axial space variable, $t$ : time variable, $m^{\prime}$ : mass per unit length, $r$ : damping per unit length.) Assuming a time dependency $e^{j \omega t}$, the time variable is transformed to the frequency domain and thus, the differential equation for steady-state oscillations is:

$$
E I \frac{\partial^{4} U_{z}(x, \omega)}{\partial x^{4}}+\left(-\omega^{2} m^{\prime}+j \omega d^{\prime}\right) U_{z}(x, \omega)=0
$$

The effect of actuation forces and the forces resulting from the liquid loading on the $U$ shaped structure's ends are considered in the boundary conditions (assuming no bending moments at the end of the beam):

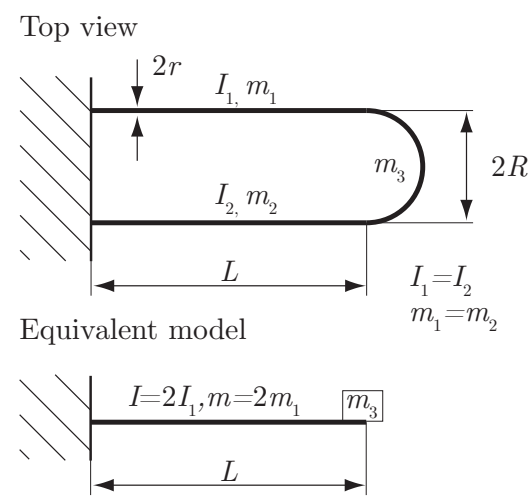

Fig. 5. Model for the analytical description of the Ushaped cantilever

$$
\begin{array}{rlll}
U_{z}(0, \omega) & =0 & & \frac{\partial U_{z}(0, \omega)}{\partial x}=0 \\
\frac{\partial^{2} U_{z}(L, \omega)}{\partial x^{2}}=0 & \frac{\partial^{3} U_{z}(L, \omega)}{\partial x^{3}}=-\frac{1}{E I} \sum_{i} F_{\text {end }, i}
\end{array}
$$

where $F_{\text {end }, i}$ denotes all forces acting on the end of the U-shaped cantilever.

\subsection{Modeling of the viscosity sensors}

To account for the fluid structure interaction of the two straight arms of the U-shaped beam and the variants of its ends (cylinder, platelet, sphere) the following modeling approaches for oscillating cylinders, platelets and spheres are pursued.

For these three cases the fluid forces consist of an inertial and a dissipative part and thus can be split up in the form:

$$
F_{F}^{\left({ }^{\prime}\right)}(x, \omega)=-\omega^{2} m_{f}^{\left({ }^{\prime}\right)}(\omega) U_{z}+j \omega d_{f}^{\left({ }^{\prime}\right)}(\omega) U_{z}
$$

where a prime denotes a quantity per unit length. For considering the effect of the liquid on the deflection of the arms which is described by Eq. (1), the parameters $m^{\prime}$ and $d^{\prime}$ are split up as follows

$m^{\prime}=m_{0}^{\prime}+m_{f}^{\prime} \quad$ and $\quad d^{\prime}=d_{0}^{\prime}+d_{f}^{\prime}$

into the beam's intrinsic parameters (denoted by the subscript 0 ) and the parameters resulting from the liquid loading (denoted by the subscript $f$ ). There, $m_{0}^{\prime}=\rho A$ (where $\rho$ is the beam's mass density and $A$ its cross section) and $d_{0}^{\prime}$ is determined through calibration measurements.

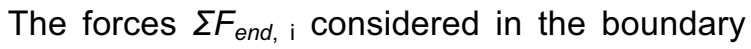
conditions Eq. 3 are:

$\sum_{i} F_{\text {end }, i}=F_{L}-\left(-\omega^{2} m_{\text {end }}+j \omega d_{\text {end }}\right) U_{z}(L, \omega)$

with the actuating Lorentz forces $F_{\mathrm{L}} \approx I_{\text {in }} B 2 R\left(l_{\text {in }}\right.$ is the amplitude of the excitation current, c.f. Fig. $3, B$ is the magnetic flux density provided by a permanent magnet, c.f. Fig. 1 and $2 R$ is the U's width, c.f. Fig. 5). The parmeters $m_{\text {end }}$ and $d_{\text {end }}$ in Eq. 6 calculated as follows:

$m_{\mathrm{end}}=m_{3}+m_{f, i} \quad$ and $\quad d_{\mathrm{end}}=d_{f, i}$

where $m_{3}$ is the lumped mass of the cantilever's end (mass of cylinder, platelet, sphere) and $m_{\mathrm{f}, \mathrm{i}}$ and $d_{f, i}$ are the parameters from the liquid loading of the particular case (cylinder, platelet, sphere).

1. Oscillating Cylinder: The fluid forces per unit length on an oscillating cylinder surrounded by a liquid with dynamic viscosity $\mu_{\mathrm{f}}$ and mass density $\rho_{\mathrm{f}}$ are $[13,14,15]$ : 


$$
\begin{gathered}
F_{F}^{\prime}(x, \omega)= \\
\pi \rho_{f} \omega^{2} r^{2}\left(1-\frac{4 j K_{1}(j \sqrt{-j R e})}{\sqrt{-j R e} K_{0}(j \sqrt{-j R e})}\right) U_{\mathrm{z}}(x, \omega) \\
\text { where } \quad R e=\frac{\rho_{f} \omega r^{2}}{\mu_{f}}
\end{gathered}
$$

( $r$ : wire's radius, $K_{0}, K_{1}$ : modified Bessel functions.) With this, the parameters $m_{f}^{\prime}$ and $d_{f}^{\prime}$ from Eqs. 4 and 5 can be expressed for the case of an oscillating cylinder in closed form through Taylor series expansion as follows:

$$
\begin{aligned}
m_{f, c}^{\prime}=m_{f}^{\prime} & =\pi \rho_{f} r^{2}\left(1+\frac{4}{\sqrt{2 R e}}\right) \text { and } \\
d_{f, c}^{\prime}=d_{f}^{\prime} & =\pi \rho_{f} \omega r^{2}\left(\frac{4}{\sqrt{2 R e}}+\frac{123}{64 R e}\right)
\end{aligned}
$$

For a straight end of the $U$ (unlike as in Fig. 5) the parameters $m_{\mathrm{f}, \mathrm{i}}$ and $d_{\mathrm{f}, \mathrm{i}}$ from Eq. 7 are:

$$
m_{f, c}=2 R m_{f, c}^{\prime} \quad \text { and } \quad d_{f, c}=2 R d_{f, c}^{\prime}
$$

2. Oscillating Platelet: The fluid forces $F_{\mathrm{F}, \mathrm{p}}$ acting on an infinitely thin, oscillating platelet with surface $A_{p}$ are:

$F_{\mathrm{F}, \mathrm{p}}=2 T_{f} A_{p}$

where $T_{f}$ are the shear stresses resulting from the liquid loading acting at the solidliquid interface. These shear stresses are modeled assuming one dimensional shear wave propagation in the liquid and thus [4, 10]:

$$
T_{f}=U_{\mathrm{z}}(L, \omega)(1-j) \sqrt{\frac{\mu_{f} \rho_{f} \omega^{3}}{2}}
$$

with this and taking Eqs. 4 and 12 into account, it follows for the platelet that:

$$
m_{f, p}=A_{p} \sqrt{\frac{\mu_{f} \rho_{f}}{2 \omega}} \quad \text { and } \quad d_{f, p}=A_{p} \sqrt{\frac{\mu_{f} \rho_{f} \omega}{2}}
$$

3. Oscillating Sphere: In [16] the fluid forces acting on a sphere, oscillating with velocity $v_{\mathrm{s}}(t)$ are given as follows:

$$
\begin{aligned}
F_{F, s}= & 6 \pi \mu_{f} r_{s}\left(1+\frac{r_{s}}{\delta}\right) v_{s}(t) \\
& +3 \pi r_{s}^{2} \sqrt{\frac{2 \mu_{f} \rho_{f}}{\omega}}\left(1+\frac{2 r_{s}}{9 \delta}\right) \frac{\partial v_{s}(t)}{\partial t}
\end{aligned}
$$

with the penetration depth

$$
\delta=\sqrt{\frac{2 \mu_{f}}{\omega \rho_{f}}}
$$

and thus, taking Eqs. 4 and 15 into account, for an oscillating sphere, it follows that:

$$
\begin{aligned}
d_{f, s} & =6 \pi \mu_{f} r_{s}\left(1+\frac{r_{s}}{\delta}\right) \text { and } \\
m_{f, s} & =3 \pi r_{s}^{2} \sqrt{\frac{2 \mu_{f} \rho_{f}}{\omega}}\left(1+\frac{2 r_{s}}{9 \delta}\right)
\end{aligned}
$$

\subsection{Modeling of the mass density sensor}

In a first approach for modeling the mass density sensor liquid damping is assumed to be negligible and the same governing equations as for the viscosity sensor are used. The differences from the modeling of the latter are as follows: The second moment of area of a beam with circular cross-section in Eq. 1 is replaced by the second moment of area of a ring. The parameters $m^{\prime}$ and $d^{\prime}$ are split up as follows:

$m^{\prime}=\left(r_{o}^{2}-r_{i}^{2}\right) \pi \rho+r_{i}^{2} \pi \rho_{f} \quad$ and $\quad d^{\prime}=d_{0}^{\prime}$

where $r_{\mathrm{o}}$ and $r_{\mathrm{i}}$ are the tube's outer in inner radii. In this simplified case $d^{\prime}{ }_{0}$ accounts for the tubes intrsic damping as well as for damping resulting from the ambient air. The forces $\Sigma F_{\text {end }, \mathrm{i}}$ considered in the boundary conditions Eq. 3 are:

$\sum_{i} F_{\mathrm{end}, i}=F_{L}+\omega^{2} m_{\mathrm{end}} U_{z}(L, \omega)$

With Eqs. 2 to 19 the steady-state amplitudes of the deflection $U_{z}(x, \omega)$ for the viscosity and the mass density sensor can be derived.

\subsection{Modeling of acutation and readout}

The equivalent electrical circuit for modeling the measured output voltage Vout is shown in Fig. 3. With this, (and neglecting the $10 \mathrm{M} \Omega$ input resistance to the lock-in amplifier) the input current $I_{\text {in }}$ necessary for Lorentz force actuation is and Vout can be given as follows:

$$
I_{\text {in }}=\frac{V_{g}}{50 \Omega+R_{s}+R_{w}} \quad \text { and } \quad V_{\text {out }}=V_{\mathrm{M}}+I_{\text {in }} R_{w}
$$

There, the motion induced Voltage $V_{M}$ considering a straight end of the U-shaped cantilever is:

$V_{\mathrm{M}}=j \omega 2 R B U_{z}(L, \omega)$

Figure 6 shows the results for the frequency responses for nominal values: $V_{g}=0.1 \mathrm{~V}$, $R_{\mathrm{s}}=1 \mathrm{k} \Omega, R_{\mathrm{w}}=0.2 \Omega, L=15 \mathrm{~mm}, 2 R=8 \mathrm{~mm}$, $\rho=19.3 \mathrm{~g} / \mathrm{cm}^{3}, E=411 \mathrm{GPa}, \quad r=200 \mu \mathrm{m}$ and $B=0.7 \mathrm{~T}$ and viscosities for Acetone, Methanol, Ethanol and Isopropanol at $25^{\circ} \mathrm{C}$ : [0.21, 0.47, $1.03,2.05] \mathrm{mPas}$. These results are relatively good agreement with first measurement results see Figure 9.

Figure 7 shows a comparison for relative values for quality factors and resonance frequencies for the viscosity sensors (immersed type) with 

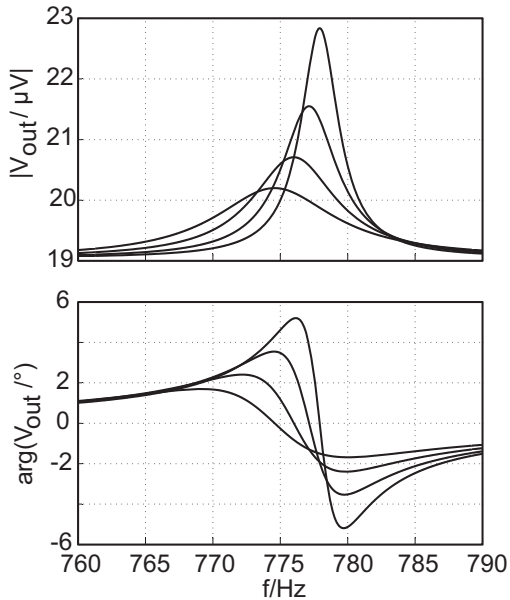

Fig. 6. Theoretical results for the frequency responses for nominal values.

different ends (straight end, plate and sphere) for varying viscosities and mass densities. (In case of varying viscosities, the mass density was set to $1 \mathrm{~g} / \mathrm{cm}^{3}$ and in case of varying mass densities, the viscosity was set to $1 \mathrm{mPas}$.) There, it can be observed, that the shape of the end does not have a significant effect on the relative change of the quality factor but on the resonance frequency for varying mass densities. However, a noteworthly effect of the U's end shape can be observed in the relative change of the resonance frequency for varying mass densities. In fact, an attached sphere increases the sensitivity to mass density, where an attached platelet would yield in a decrease of sensitivity to mass density.
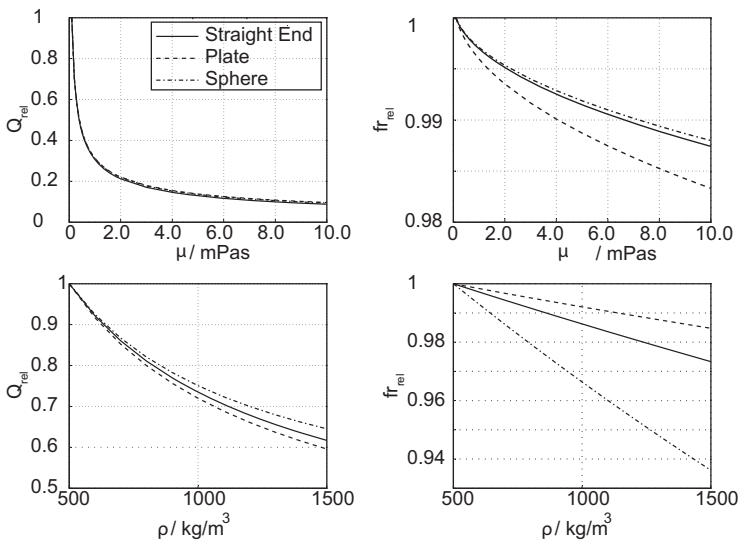

Fig. 7. Relative changes for the Quality factor and resonance frequency for varying viscosities and mass densities.

\section{Measurements}

First measurements were made with prototypes for the viscosity and mass density sensor, see Figs. 8, 9 and 10. The viscosity sensor consists of a U-shaped, $400 \mu \mathrm{m}$ thick tungsten wire surrounded by the sample liquid. The mass density sensor is made of a stainless steel tube (with outer and inner diameters of 0.8 and

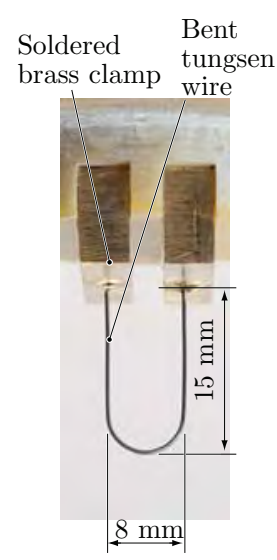

a) Wire-based viscosity sensor

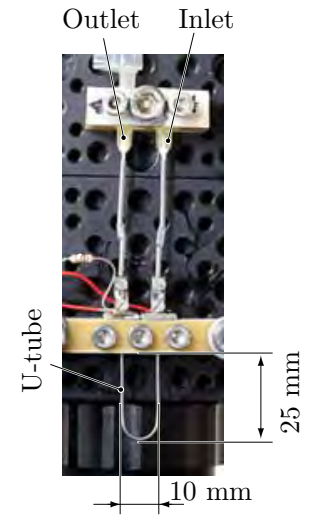

b) Mass density sensor

Fig. 8. First prototypes for viscosity and mass density sensor.

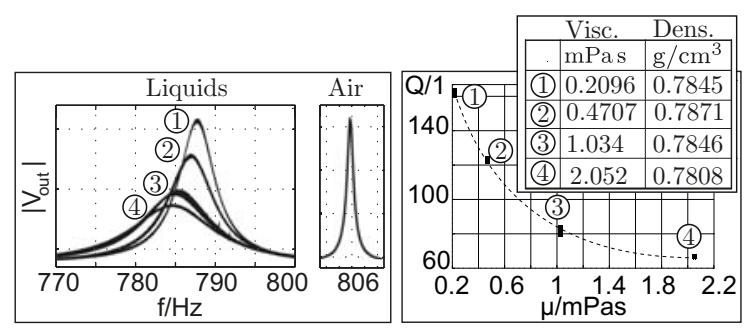

Fig. 9. Evaluation of viscosity measurements.

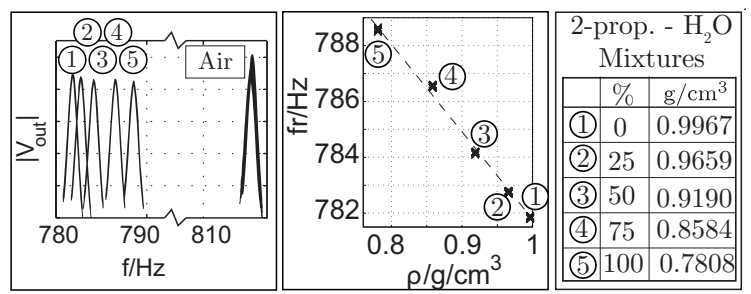

Fig. 10. Evaluation of mass-density measurements.

$0.5 \mathrm{~mm}$ respectively) carrying the sample liquid inside. All of these devices yielded excellent long term stability i.e. good repeatability of the measurements as well as low cross-sensitivities to temperature, compared to devices consisting of straight, doubly clamped structures, c.f., [10]. The low dependency of the resonance frequency to temperature is obtained by the singly clamped design approach itself and the choice of the used materials (tungsten) featuring a low thermal expansion coefficient.

Scaling experiments were performed to investigate the achievable range of resonance frequencies. For this, three different widths (2R) have been investigated at different structure lengths $L$ for the case of $400 \mu \mathrm{m}$ thick tungsten wires, see Fig. 10. The resonance peak $d V$ of the motion-induced voltage for the resonance at $30 \mathrm{kHz}$ is $2 \mu \mathrm{V}$. This shows the ability for obtaining an even higher range of resonance frequencies, as the resolution of conventional for the particular case of lock-in amplifiers is in 


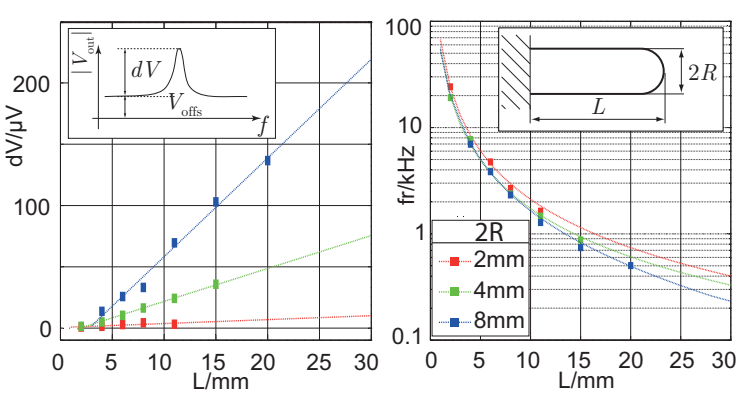

Fig. 10. Evaluation from scaling experiments.

the nano Volt range. I.e., for the particular case of $400 \mu \mathrm{m}$ thick tungsten wires, resonance frequencies of $100 \mathrm{~Hz}$ to $100 \mathrm{kHz}$ can be obtained with device lengths from $1 \mathrm{~mm}$ to 3 $\mathrm{cm}$.

\section{Conclusion and Outlook}

The choice of U-shaped, wire based resonators which can be considered as singly clamped beams, yields an excellent improvement of measurements stability, reliability and furthermore the cross-sensitivity to temperature is drastically decreased, compared to straight wires, i.e., doubly clamped beams. A first modeling approach for the presented mass density and viscosity sensors shows good agreements with measurements achieved with first prototypes. A (geometrically) scalingexperiment shows that the desired frequency range of $100 \mathrm{~Hz}$ to $100 \mathrm{kHz}$ can be covered with these devices.

Regarding future work, the theoretical findings for the variants of the U-shaped device with attached objects have to be confirmed by experimental verification. Furthermore the analytical model for the mass density and the viscosity sensor have to be extended by taking into account the rounded end (instead of the straight end, as presented in this contribution). For the modeling of the mass density sensor (tube) the effect of viscosity on the resonance frequency hast to be investigated. Finally, an extensive measurement series has to be performed enabling the calibration of the sensors and estimation of achievable accuracies of the devices.

\section{Acknowledgment}

We are indebted to the Austrian Centre of Competence in Mechatronics (ACCM) for the financial support. We also want to thank Bernhard Mayrhofer and Johann Katzenmayer for their help and excellent assistance.

\section{References}

[1] C. Tropea et al., Springer Handbook of Experimental Fluid Mechanics,Springer-Verlag, Berlin Heidelberg, 2007
[2] C.W. Macosko, Rheology, Principles, Measurements and Applications, Wiley- $\mathrm{VCH}$, 1994

[3] D.S. Viswanath, T.K. Ghosh, D.H.L. Prasad, N.V.K. Dutt, and K.Y. Rani. Viscosity of Liquids. Theory, Estimation, Experiment and Data. Springer, 2007.

[4] S. J. Martin, V. E. Granstaff, and Gregory C. Frye. Characterization of a quartz crystal microbalance with simultaneous mass and liquid loading. Anal. Chem., volume 63, pp. 2272-2281, 1991.

[5] C. Riesch, Micromachined Viscosity Sensors, Shaker Verlag, 2009.

[6] M. Heinisch, E.K. Reichel, I. Dufour and B. Jakoby, A resonating rheometer using two polymer membranes for measuring liquid viscosity and mass density, Sens. Actuators A: Physical 172 (2011) 82-87, doi: 10.1016/j.sna.2011.02.031

[7] E. K. Reichel et al., Modeling of the fluid-structure interaction in a fluidic sensor cell, Sens. Actuators A 156 (2009) 222-228, doi:10.1016/j.sna.2009.03.002.

[8] E. K. Reichel et al., Analysis and experimental verification of a metallic suspended plate resonator for viscosity sensing, Sensors and Actuators A: Physical, 162/2, 2010, pp 418-424

[9] M. Heinisch, E.K. Reichel, and B. Jakoby. A suspended plate in-plane resonator for rheological measurements at tunable frequencies. In Proc. Sensor + Test, pages 6166, 2011.

[10] M. Heinisch, E.K. Reichel, I. Dufour, and B. Jakoby. Tunable resonators in the low khz range for viscosity sensing. Sensors and Actuators A: Physical 186 (2012) 111-117, doi: 10.1016/j.sna.2012.03.009

[11] W.W. Weaver, S.P. Timoshenko, and D.H. Young. Vibaration Problems in Engineering. Wiley, 5th edition, 1990.

[12] F. Keplinger, R. Beigelbeck, F. Kohl, S. Kvasnica, A. Jachimowicz and B. Jakoby. Frequency and transient analysis of micromachined U-shaped cantilever devices for magnetic field measurement, Solid-State Sensors, Actuators and Microsystems, pp. 630-635, 2005

[13] L.D. Landau and E.M. Lifshitz. Fluid Mechanics. Butterworth-Heinemann, 1987.

[14] L. Rosenhead. Laminar Boundary Layers. Clarendon, Oxford, 1963.

[15] J.E. Sader. Frequency response of cantilever beams immersed inviscous fluids with applications to the atomic force microscope. Journal of Applied Physics, 84: 64 - 76, 1998. 